\title{
Visibilidade da produção científica de grupos de pesquisa em serviço social do estado do Rio de Janeiro
}

\author{
Visibility of scientific production of social \\ work research groups in the federal \\ state of Rio de Janeiro, Brazil
}

\author{
Tânia CHALHUB' \\ Claudia GUERRA²
}

\section{Resumo}

Pesquisa descritiva quantitativa de grupos de pesquisa em Serviço Social de universidades do Estado do Rio de Janeiro cadastrados no Conselho Nacional de Desenvolvimento Científico e Tecnológico que têm por objetivo analisar visibilidade da produção científica dos líderes desses grupos. A visibilidade dos resultados de pesquisas em Serviço Social viabilizaria a ampliação da cidadania. Foi realizado levantamento da produção na plataforma Lattes e analisado o período de 2003 a 2010. Dentre os periódicos mais utilizados por esses grupos, que representam boa parte da produção científica em Serviço Social no país, a maioria está veiculada pela forma tradicional de comunicação, publicação impressa, até 2009. Desses periódicos alguns são reconhecidos por sua abrangência nacional, porém os que apresentam maior número de artigos publicados pelo grupo estudado têm sua visibilidade comprometida por serem impressos ou por não estarem indexados em bases de dados de acesso via web. O padrão de comunicação científica corrobora o de outras áreas das Ciências Sociais, contudo não apresenta boa visibilidade como outras áreas que dispõem de número expressivo de periódicos de acesso livre e indexação em bases de dados (Science Citation Index), diretórios (Latindex) e redes cooperativas (Redalyc).

Palavras-chave: Comunicação científica. Pesquisa em serviço social. Pesquisadores acadêmicos. Periódico científico.

\footnotetext{
Abstract

This work describes a quantitative research into the visibility of the scientific production of the leaders of Social Work research groups in the public universities of the state of Rio de Janeiro, registered with the Conselho Nacional de Desenvolvimento Científico e Tecnológico (Brazilian National Council for Scientific Research). The visibility of the results of Social Work research makes possible the exercise of rights and the broadening of the concept of citizenship. A search was conducted of the scientific production registered on the Lattes platform (a Brazilian scientific database) between 2003 and 2010. The best part of these articles in periodical journals is composed of the traditional form of communication, namely printed documents; amongst these, a good number are not indexed by web accessed databases, which has an adverse impact on visibility. This pattern of scientific communication corroborates the typical pattern found in other Social Work areas. In terms of visibility, this group does not match the greater visibility of other areas that have a larger number of open access journals, as well as periodicals that make use of indexing channels such as databases (Science Citation Index), directories (Latindex) and cooperative networks (Redalyc).

Keywords: Scientific communication. Research into social work. Scientific researcher. Scientific journals.

1 Pós-doutorando em Ciência da Informação, Instituto Brasileiro de Informação em Ciência e Tecnologia. R. Lauro Müller, 455, 22290-160, Rio de Janeiro, RJ, Brasil. Correspondência para/Correspondence to: T. CHALHUB. E-mail: <chalhubtania@yahoo.com.br>

2 Doutoranda em Ciência da Informação, Instituto Brasileiro de Informação em Ciência e Tecnologia. Rio de Janeiro, RJ, Brasil.

Recebido em 22/4/2011, reapresentado em 7/7/2011 e aceito para publicação em 24/8/2011.
} 


\section{Introdução}

A ciência não se faz em silêncio. Do interior dos laboratórios, museus, universidades e gabinetes de grupos de pesquisa emerge o conhecimento científico, decorrente de incansáveis observações empíricas e pesquisas, algo que só se torna efetivo quando é publicado. A socialização da informação possibilita a renovação do conhecimento através de novos impulsos para a geração de novas pesquisas, além da disseminação desse conhecimento para aplicação e transformação da realidade. Segundo Meadows (1999), a necessidade de acumular dados, desenvolver teorias e experiências simultaneamente e modificar ideias faz com que os cientistas se envolvam com a comunicação. Nesse sentido, à pesquisa científica é conferido o aspecto social por meio da troca de informação entre os pares. É na comunicação que a ciência se torna um fato social.

A partir da década de 1990, com o advento das novas tecnologias de redes de comunicação, a produção intelectual encontrou novas formas de colaboração e compartilhamento que alteraram as relações entre trabalho e pesquisa. González de Gómez (2003) aponta como condição emergente e significativa na atividade científica a demanda de "prestação de contas" (accountability):

A 'prestação de contas' difere do éthos científico, já que o éthos é definido como responsabilidade individual dos pesquisadores ou como atributo abstrato de uma esfera ideal da ciência, e a'prestação de contas' refere-se a uma figura eminentemente social, ancorada em conjuntos de atores e instituições que assumem responsabilidade pelas potências inovadoras em campos específicos da vida coletiva. A pesquisa ficaria logo sujeita às demandas de resultados, visibilidade, acuidade e transparência, vindas dos sujeitos e domínios sociais em que adquire relevância e pertinência (González de Gómez, 2003, p.71).

Prestar contas do conhecimento se insere na perspectiva de um novo éthos científico no que se refere ao entrelaçamento de novas redes sociais de produção e comunicação, ainda aqui relacionado com o tradicional propósito da comunicação científica.

Dentre as normas sociais estabelecidas por Merton relativas à conduta dos cientistas, o sentido de comunidade (communality) reflete o requisito de que o conhe- cimento científico deve ser de propriedade comum (Meadows, 1999). Para que isso se tornasse possível, canais de divulgação e disseminação de informações científicas foram criados simultaneamente ao processo de desenvolvimento da ciência e, como processo dinâmico, evoluiu consideravelmente, principalmente no Século XX com o advento das tecnologias da informação. Os canais de informação podem ser comunicações formais - livros, periódicos impressos ou eletrônicos e anais de conferências e congressos; e informais - redes humanas diversas tais como: cartas, e-mails, chats, e encontros sociais nos eventos.

A comunicação entre pares se dá desde o início da pesquisa, sendo comum, durante o seu desenvolvimento, a utilização da comunicação informal e, à medida que se avança no cronograma, a comunicação de dados parciais em veículos formais. No final da pesquisa, os resultados são, em geral, publicados em periódicos científicos ou em anais dos eventos científicos ou mesmo divulgados oralmente nos eventos específicos da área. A publicação confere validação e registro desses resultados.

Na comunicação científica, o papel do pesquisador se revela em suas motivações em divulgar suas descobertas e conclusões obtidas no decorrer da pesquisa. Pode-se falar de um conjunto de práticas sociais, regras de conduta voltadas para as comunidades científicas, universidades, centros de pesquisa e agências financiadoras, naquilo que Meadows (1999, p. 80) reconhece como motivação da ordem da identidade científica "a proposição segundo a qual os membros da comunidade científica tendem tacitamente a concordar com um conjunto de normas, que devem, em princípio, orientá-las sobre a forma como atua e se comunica".

Uma das principais normas é a necessidade de atestar a produtividade individual do pesquisador por meio da prática comunicativa: em artigos publicados em revistas de renome e comunicações apresentadas em eventos oficiais da área. Essa prestação de contas de suas atividades científicas possibilita ao pesquisador o reconhecimento pelos pares e o capacita para novos empreendimentos financiados pelas universidades e agências de fomento. Para a maioria das áreas do conhecimento as revistas indexadas ainda se constituem como o centro do sistema tradicional de comunicação científica por serem os canais preferenciais, ainda que imperfeitos 
para a certificação do conhecimento científico. Contudo, os eventos científicos também representam um importante canal de comunicação científica, possibilitando interação entre seus participantes, podendo ainda, além da troca, oferecer o registro dos trabalhos apresentados com a sua publicação em anais, seja da íntegra ou dos resumos. Em algumas áreas, como a Medicina, é comum os anais serem depositados em repositórios institucionais ou publicados em periódicos científicos em exemplares especiais, como o fascículo de novembro 2010 da Blood, periódico científico da American Society of Hematology (v.116, n.21).

A proposta desta pesquisa - analisar a visibilidade da produção científica da área no contexto do acesso livre - é pertinente, dado o avanço das publicações eletrônicas nas duas últimas décadas do Século XX, que presenciaram a consolidação das ações e políticas brasileiras de incentivo ao desenvolvimento científico; avanço que também possibilitou a inserção do Serviço Social no panorama científico nacional, principalmente através de incentivo às publicações científicas dos programas de pós-graduação stricto sensu.

Apesar de grandes avanços em relação à pesquisa em Serviço Social, com importantes análises sobre as temáticas, há ainda a realizar a evolução histórica da construção do conhecimento, os principais desafios, as questões metodológicas relacionadas, em especial, à divulgação desse conhecimento. Qual é a visibilidade da produção dos líderes de pesquisa da área? Este trabalho propõe-se a analisar a visibilidade da produção científica do líder de pesquisa em Serviço Social.

\section{A visibilidade da produção científica}

A produção científica e sua divulgação, através de publicações em periódicos científicos indexados, tiveram um aumento exponencial no Brasil nas últimas duas décadas do Século XX. Esse crescimento pode ser creditado à ampliação de oferta de bolsas de fomento à pesquisa, incentivo para criação e manutenção de publicações científicas, consolidação de sistemas de avaliação de méritos nas universidades, entre outras.

Essas não foram, porém, as únicas mudanças na comunidade científica brasileira no final do Século XX. O Brasil também aderiu ao novo paradigma da comunicação científica iniciado em 2001, com o movimento de acesso livre à informação científica. Esse movimento originado de questões intelectuais e políticas que relacionam a informação como um bem público dado que, geralmente, é resultante de pesquisa científica financiada com recursos públicos. Dessa forma, as publicações daí resultantes devem ser disponibilizadas em plataformas de acesso aberto não só para profissionais ligados a essa atividade intelectual mas também para o público em geral.

O advento do periódico eletrônico, fruto do movimento de acesso livre, potencializou o acesso aos conteúdos das pesquisas, colocando em xeque o sistema de publicação científica tradicional, no qual os direitos autorais são detidos por editores comerciais que "atribuem preços excessivos e impõem barreiras de permissão sobre publicações de resultados de pesquisas financiadas com públicos, limitando a visibilidade e a circulação do conhecimento científico" (Leite, 2009, p.14). Segundo Castro (2006, p.60), o movimento de acesso livre provocou uma reestruturação do fluxo da comunicação científica, "além da publicação eletrônica de documentos, incluindo a adoção de transformações nos padrões de comportamento da comunidade científica e sua relação com a sociedade", assim como superando barreiras de visibilidade e acesso à literatura.

Nesse contexto, publicações em acesso livre, dentre outros aspectos, representam a democratização do acesso à informação científica devido à ampliação do número de pesquisadores, estudantes e até leigos que podem alcançar o conhecimento científico, naquilo que se define como visibilidade (Zimba; Mueller, 2004, p.49):

[...] como grau de exposição e evidência de um pesquisador frente à comunidade científica. Uma posição de visibilidade alta é aquela na qual os trabalhos e ideias do pesquisador são facilmente acessíveis. Sendo acessíveis, poderão ser recuperados, lidos e citados. [...] em resumo, quanto maior o grau de visibilidade, maiores são as chances do pesquisador de ser lido, avaliado e citado. As chances de um autor para aumentar sua visibilidade são então dependentes de sua maior exposição à comunidade mundial de pares e isso tem mais probabilidade de ocorrer quando a publicação de trabalhos de pesquisa se dá em periódicos internacionais, principalmente aqueles indexados em bases de dados especializadas e internacionais, de ampla divulgação e prestígio.

TransInformação, Campinas, 23(3):185-194, set./dez., 2011 
A visibilidade da produção científica foi potencializada com a adesão ao acesso livre de diversas publicações eletrônicas, através da indexação em bases eletrônicas como Web of Knowledge, MedLine e diretórios de publicações científicas (ferramentas que permitem localização dos documentos). Apesar de não ter a dimensão de bases como o Institute Scientific Information (ISI), a inclusão do periódico na Scientific Eletronic Library Online (SciELO), cujos critérios de seleção são baseados em padrões internacionais, também oferece visibilidade aos títulos ali indexados.

Em algumas áreas como a Medicina, a visibilidade das publicações brasileiras já é reconhecida, tanto nacional quanto internacionalmente, com periódicos indexados no ISI Web of Knowledge entre outras bases. Por outro lado, existem áreas como o Serviço Social com padrão de comunicação científica que apresenta outra tendência: publicações em anais de congressos e livros equivalem a 78,4\% do total da produção, enquanto artigos em pe-riódicos representam somente 21,5\% da produção cientí-fica de pesquisadores brasileiros (Chalhub; Oliveira, 2010). Da publicação de artigos, a maioria não se encontra em periódicos de acesso livre e indexação em bases de dados especializados e internacionais.

A pesquisa em Serviço Social no Brasil tem contribuído de forma expressiva para a construção e consolidação de conhecimento específico da área e sua divulgação possibilita a reflexão sobre a intervenção profissional numa relação dialética entre investigação e intervenção críticas na realidade social. Segundo Bourguignon (2007, p.47) a pesquisa nessa área tem contribuído para avanços:

Em diferentes campos da ação profissional, no âmbito das políticas públicas, no enfrentamento das expressões da questão social, na construção da proposta curricular e definição dos seus fundamentos teóricos e metodológicos, na consolidação do projeto ético-político profissional, entre outros aspectos.

Tal construção se expressa em reflexões teóricas importantes, em elaborações conceituais e compreensão crítica da realidade, principalmente das questões políticas e sociais, possibilitando um fortalecimento da base científico-profissional, além de transformações na realidade.
Um aspecto importante para os avanços na pesquisa em Serviço Social foi o reconhecimento por órgãos como Conselho Nacional de Desenvolvimento Científico e Tecnológico (CNPq), Fundação de Amparo à Pesquisa do Estado do Rio de Janeiro (Faperj) e Fundação de Amparo à Pesquisa do Estado de São Paulo (Fapesp) do Serviço Social como área de conhecimento, o que incentivou pesquisas em diversos núcleos de pós-graduação strictu senso e o desenvolvimento de grupos de pesquisas, com uma produção significativa de trabalhos (Sposati, 2007).

A inserção da pesquisa na formação profissional foi um instrumento fundamental para a profissão em Serviço Social, tanto para docência quanto para a prática profissional. Vale aqui um parêntese sobre a formação profissional, que teve início no Brasil nas primeiras décadas do Século XX com as escolas de Serviço Social de São Paulo, em 1936, e do Rio de Janeiro, em 1937. Porém, a produção de conhecimento nessa área no país iniciou-se, de forma tardia, na década de 1970, com a criação dos cursos de pós-graduação. As Ciências Sociais, de uma forma geral, tiveram a expansão da pesquisa na década de 1960 (Kameyama, 1998).

A análise da produção do conhecimento em Serviço Social foi objeto de pesquisa de diversos autores que estudaram a produção acadêmica dos docentes (pesquisas) e dos discentes (dissertações e teses) com relação às temáticas pesquisadas, metodologias utilizadas, programas de pós-graduação e suas linhas de pesquisa, resultando em inúmeros artigos e livros (Baptista, 1992; Carvalho, 1992; Kameyama, 1998; Simionatto, 2005; Yasbeck, 2005; Sposati, 2007).

Esses estudos discutem a importância da relação da prática investigativa na formação e na atuação profissional, além do seu impacto na realidade social e na elaboração de políticas públicas. Porém, o processo de construção do conhecimento se completa com a comunicação desse conhecimento aos profissionais que necessitam realimentar a prática e o próprio conhecimento. Percebe-se que, apesar de haver uma grande produção de pesquisas na área, sua tendência é por publicação não periódica, e a minoria de artigos encontra-se publicada em periódicos e, ainda que estejam disponibilizados em repositórios digitais, "a revista com maior número de 
trabalhos publicados, a mais representativa da área tem sua edição em entidade comercial (Editora Cortez) e não possui o perfil ${ }^{3}$ de acesso livre" (Chalhub; Oliveira, 2010).

Vale ressaltar que essa é uma situação de uma área específica, Serviço Social, que assim se apresenta no Brasil, mas que em outros países, nos EUA, por exemplo, mostra outra configuração com relação a publicações científicas.

\section{Métodos}

Foi realizada uma pesquisa quantitativa descritiva, abordando a produção de pesquisadores do Estado do Rio de Janeiro com grupos de pesquisa registrados no CNPq e utilizando a Plataforma Lattes do mesmo órgão para identificação da produtividade dos líderes dos grupos de pesquisa. Os critérios de análise foram baseados na definição de visibilidade adotada por Mueller (Zimba; Mueller, 2004), utilizando o indicador publicações de autores em periódicos indexados ${ }^{4}$ acrescido do indicador disponibilização na Internet do texto completo, de Oliveira (2005).

Também foi feito um levantamento no Diretório de Grupos de Pesquisa do CNPq no site do órgão federal de fomento à pesquisa. A busca pelos grupos se processou por unidade da federação, grandes áreas (Ciências Sociais Aplicadas) e área (Serviço Social).

Uma vez identificados, foi realizada uma busca no sistema eletrônico do CNPq para localizar o curriculum vitae, na Plataforma Lattes, de cada um dos líderes dos grupos de pesquisa de unidades de ensino superior do Estado do Rio de Janeiro, identificando sua produção entre 2003 e 2010, publicada em periódicos científicos. A opção pelos grupos de pesquisa do Estado do Rio de Janeiro se deve ao fato de ser a unidade federativa com maior número de grupos registrados no CNPq, 37 num total de 172, e ter entre seus líderes autores de reconhecida contribuição para a construção do conhecimento científico na área desde a década de 19705.

Em seguida foi feito o registro em instrumento desenvolvido para organização dos dados dos líderes dos grupos e a análise das formas de divulgação. O instrumento era composto de itens de identificação do número do grupo no registro do CNPq, instituição de afiliação do grupo (unidades de ensino superior ou instituto de pesquisa), tipo de publicação (artigo em periódicos), nome dos líderes e pesquisadores. Outro instrumento foi elaborado para o registro das informações sobre as publicações, título do periódico, local da editoração (instituição e cidade) e tipo de edição (impressa ou eletrônica).

As unidades de ensino superior às quais os grupos pertencem são Pontifícia Universidade Católica do Rio de Janeiro - (PUC-Rio) (quatro grupos), Universidade Estadual do Rio de Janeiro - (UERJ) (11 grupos), Universidade Federal Fluminense - (UFF) (dez grupos), Universidade Federal do Rio de Janeiro - (UFRJ) (dez grupos), Universidade do Grande Rio - Unigranrio (um grupo) e Centro Universitário de Volta Redonda - (UniFOA) (um grupo).

Esses grupos são compostos de 49 líderes e 138 pesquisadores. Um dos grupos foi excluído por ser multidisciplinar, de unidade de ensino particular com a grande maioria de seus pesquisadores e produção na área de Direito e pouca participação do Serviço Social. Do total levantado foram, então, excluídos dois líderes, restando para análise a produção de 47 líderes.

Depois do levantamento da produção científica, foi elaboradoum cálculo simplificado para divisão em quartis que possibilitasse uma visão geral da produção por líder. Classificada essa produção, foram analisadas as publicações periódicas segundo os indicadores: indexação do periódico e disponibilização de texto completo em acesso livre na Internet.

\section{Resultados}

Nos grupos de pesquisa em Serviço Social registrados no diretório do CNPq em 2009 percebe-se uma concentração acentuada nas regiões Sudeste (72), Nordeste (49) e Sul (41) em contraste com o baixo número nas regiões Centro-Oeste (12) e Norte (6) (Figura 1).

3 Esta revista faz parte da SciELO desde meados de 2010, disponibilizando até fevereiro de 2011 somente os números referentes àquele ano, ou seja, sem a produção científica analisada nesta pesquisa.

4 Originalmente foram utilizados dois indicadores principais: publicações dos autores em periódicos indexados nas bases do ISI e citações aos artigos escritos por esses autores.

- Vale ressaltar que grupos de outros Estados também são reconhecidos pela expressiva contribuição na produção científica em Serviço Social para o Brasil e para a América Latina, porém não poderiam ser contemplados neste estudo que teve objetivo delimitado nos grupos do Estado do Rio de Janeiro. 


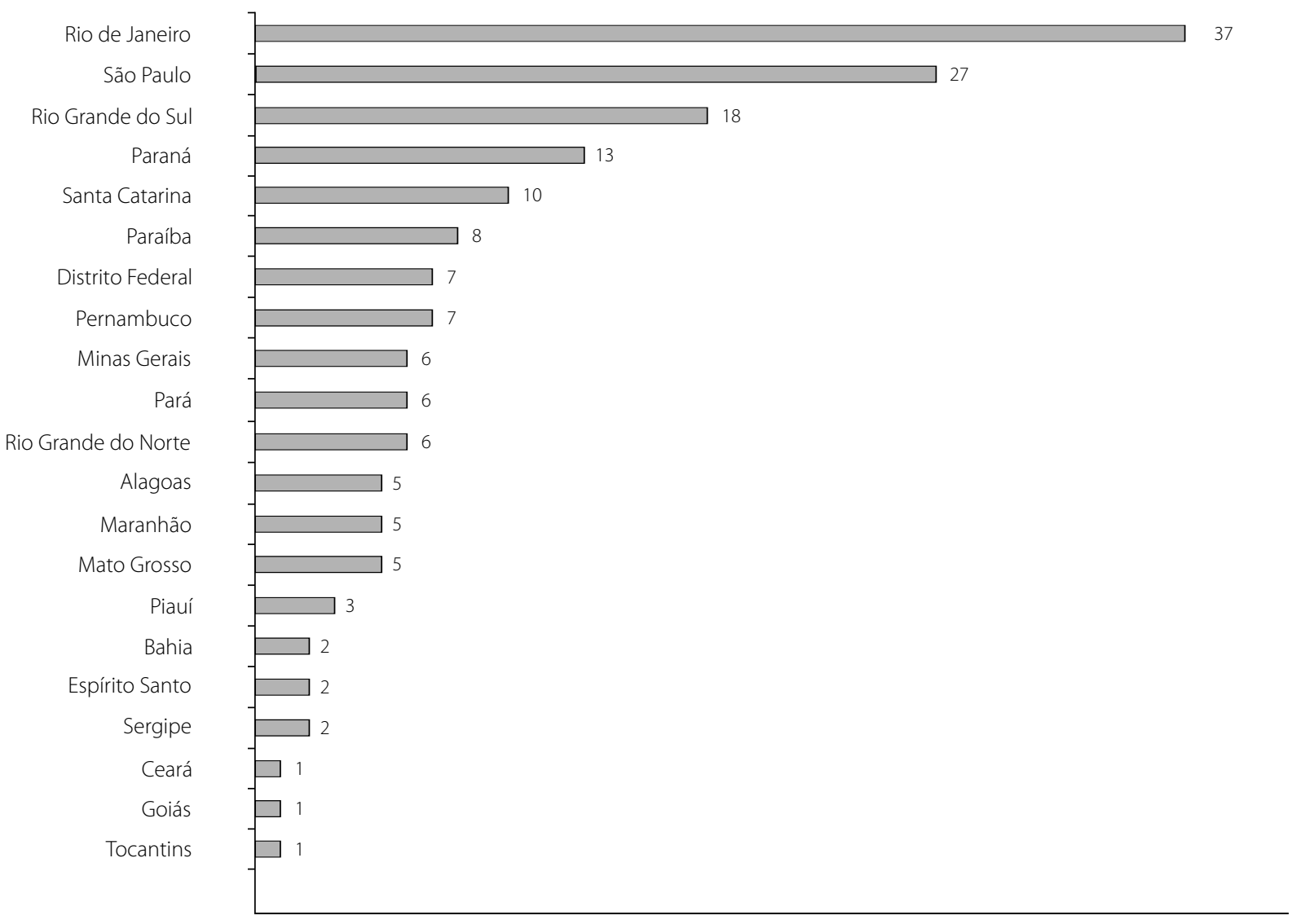

Figura 1. Grupos de pesquisa em Serviço Social registrados no Diretório de Grupos de Pesquisa do CNPq por unidade da federação em 2009. Fonte: Diretório de Grupo de Pesquisa do CNPq.

Tabela 1. Total de publicações periódicas da produção científica dos grupos de pesquisa em Serviço Social no Estado do Rio de Janeiro de 2003 a 2010 por instituição de ensino superior.

\begin{tabular}{lccc}
\hline Instituição & Grupos & Líderes & Artigos em periódicos \\
\hline PUC-Rio & 4 & 5 & 25 \\
UFF & 10 & 11 & 58 \\
UERJ & 11 & 12 & 41 \\
UFRJ & 10 & 12 & 65 \\
UNIGRANRIO & 1 & 1 & 7 \\
\hline Total & 36 & 41 & 196 \\
\hline
\end{tabular}

PUC-Rio: Pontifícia Universidade Católica do Rio de Janeiro; UFF: Universidade Federal Fluminense; UERJ: Universidade Estadual do Rio de Janeiro; UFRJ: Universidade Federal do Rio de Janeiro; UNIGRANRIO: Universidade do Grande Rio.

O Estado do Rio de Janeiro apresentou o maior número de grupos de pesquisa no Diretório de Pesquisa do CNPq, 37 grupos do total de 142. A Região Sudeste, principalmente os Estados do Rio de Janeiro e São Paulo, apresenta a maior concentração dos grupos registrados, seguida da Região Sul com distribuição equilibrada entre os três Estados. O registro de grupos de pesquisa de Pernambuco e Paraíba é também significante, colocando o Nordeste na frente da Região Centro-Oeste. A Região Norte tem o menor quantitativo de grupos registrado, 
refletindo o número de unidades de ensino com cursos de pós-graduação e pesquisadores.

NaTabela 1 são apresentados os grupos do Estado do Rio de Janeiro, que configuram a população de 41 líderes estudados, do total de 47 registrados no CNPq; não foi possível acessar o Lattes e, consequentemente, identificar a produção de seis líderes, e sua produção acadêmica publicada em canais formais de comunicação científica.

Foram analisados 196 trabalhos publicados em periódicos de 2003 a 2010. Os artigos analisados representam $18,1 \%$ dos trabalhos publicados nesse período, valor mais baixo do que os $21,5 \%$ dos artigos dos pesquisadores com bolsas de produtividade do CNPq - nível 1, no período de cinco anos (2005 a 2009) (Chalhub; Oliveira, 2010) e do que os 33,4\% de pesquisadores de Filosofia e Ciências Humanas da Universidade Federal da Bahia (Machado; Meirelles, 2005). Essa diferença na proporcionalidade do tipo de publicação é mais discrepante se comparada à diferença que Meadows (1999) preconiza nas áreas de uma forma geral, 82,0\% em Ciências e 29,0\% em Ciências Sociais.

A maioria dos artigos publicados pelos líderes de pesquisa em Serviço Social no Estado do Rio de Janeiro está em periódicos da própria área, uma pequena parcela está presente em periódicos científicos de outras áreas de conhecimento, como a Saúde Pública, Política Social, Sociologia, Psicologia e Educação.

Grande porcentagem dos periódicos identificados $(71,9 \%)$ foi utilizada para publicar somente um artigo do grupo de pesquisadores em questão, 18,7\% publicaram dois ou três artigos e 3,8\% de quatro a cinco artigos. Esses dados refletem uma dispersão dos canais de publicação do grupo, com diversidade de área dos periódicos como Revista Psicologia e Sociedade, Revista Eletrônica de História do Brasil e PHYSIS: Revista de Saúde Coletiva. Essa dispersão está também relacionada à localização das revistas de Norte a Sul do Brasil. Há ainda a participação de poucas revistas estrangeiras como as espanholas Enfermería Global e Spanish Journal of Psychology, as chilenas Revista Trabajo Social e Revista de Ciencias Sociales, as francesas Cybergeo e Espaces et Sociétés, e a argentina Revista Consciencia Social.

O Quadro 1 apresenta a lista dos principais periódi$\cos (5,5 \%)$, que foram mais utilizados (publicação de seis artigos ou mais) pelos pesquisadores como canal de comunicação de seus trabalhos, concentrando 34,5\% da produção total de artigos. Esses periódicos foram selecionados para aprofundamento dos indicadores de visibilidade.

Os periódicos com maior número de artigos publicados no período estudado também apresentam uma

Quadro 1. Principais periódicos utilizados para publicação de artigos líderes de pesquisa em Serviço Social no Estado do Rio de Janeiro de 2003 a 2010.

\begin{tabular}{llcl}
\hline Título do periódico & Tipo de edição & Qualis & Editora \\
\hline Serviço Social e Sociedade & Impressa e Eletrônica* & A 1 & Editora Cortês (SP) \\
O Social em Questão & Impressa & B 2 & Pontifícia Universidade Católica do Rio de Janeiro (PUC-Rio) \\
Ser Social & Impressa e Eletrônica & B 1 & Universidade de Brasília (UnB) \\
Temporalis & Impressa & B 1 & Associação Brasileira de Ensino e Pesquisa em Serviço Social (ABEPSS/DF) \\
Revista Katálysis & Impressa e Eletrônica & A 1 & Universidade Federal de Santa Catarina (UFSC) \\
Cadernos de Saúde Pública & Impressa e Eletrônica & A2 & Escola Nacional de Saúde Pública - Fiocruz (RJ) \\
Praia Vermelha & Impressa e Eletrônica & B 1 & Universidade Federal do Rio de Janeiro (UFRJ) \\
Revista Agora & Impressa e Eletrônica*** & A2 & Universidade Federal do Rio de Janeiro (UFRJ) \\
Em Pauta & Impressa e Eletrônica & B 1 & Universidade Estadual do Rio de Janeiro (UERJ) \\
Em Debate & Eletrônica** & B 4 & Pontifícia Universidade Católica do Rio de Janeiro (PUC-Rio) \\
Libertas On Line & Eletrônica & B 2 & Universidade Federal de Juiz de Fora - UFJF (MG) \\
Revista Sociedade em Debate & Impressa & B 1 & Universidade Católica de Pelotas \\
Textos e Contextos & A 2 & Pontifícia Universidade Católica do Rio Grande do Sul (PUCRS) \\
Revista de Políticas Públicas & Eletrônica & B 1 & Universidade Federal do Maranhão (UFMA) \\
Revista Interagir & Impressa e Eletrônica*** & B 5 & Universidade do Estado do Rio de Janeiro (UERJ) \\
\hline
\end{tabular}

"Acesso via SciELO dos números de 2010, sem a produção dos pesquisadores aqui estudados; ** Disponibilização em pdf no site da universidade; *** Acesso ao resumo do artigo. 
acentuada concentração na Região Sudeste (nove títulos) seguida das Regiões Sul (três) e Centro-Oeste (dois). Este dado não é específico da área, antes reflete o padrão de desenvolvimento científico do país como um todo, que está se alterando com os esforços das políticas de descentralização com criações de universidades públicas e institutos de pesquisas nas regiões Norte e Centro-Oeste, principalmente.

De acordo com o site da Capes (http://www.capes. gov.br/avaliacao/qualis), o Qualis de cada área expressa qual é"a potencial relevância da divulgação de trabalhos nos veículos nele incluídos para a evolução do conhecimento na área em questão". Apesar de concentrar a divulgação de estudos em anais de eventos acadêmicos, um terço dos periódicos mais utilizados pelos líderes para a divulgação das pesquisas em Serviço Social tem classificação de alta qualidade (A1 e A2), o que demonstra a preocupação em utilizar periódicos bem avaliados para dar maior visibilidade ao trabalho diante das exigências dos órgãos de fomento.

Outro aspecto que merece destaque é a presença de somente duas editoras não acadêmicas, a Editora Cortês, que publica a Revista Serviço Social e Sociedade (a mais antiga neste estudo) e a Associação Brasileira de Ensino e Pesquisa em Serviço Social (ABEPSS), que publica a Revista Temporalis. A predominância das editoras acadêmicas ou de entidades científicas também não é uma característica da área de Serviço Social, vários estudos em outras áreas já identificaram essa característica editorial dos periódicos brasileiros, latino-americanos e ibero-americanos (Chalhub; Oliveira, 2010; Oliveira et al., 2010).

Alguns dos periódicos listados no Quadro 1 têm abrangência nacional e são reconhecidos como importante canal de comunicação, não só para acadêmicos e pesquisadores como para profissionais da prática assistencial, seja pela sua história na construção de conhecimento da área e grande circulação (Serviço Social e Sociedade, Ser Social e Temporalis), seja pelo seu amplo alcance através de edição eletrônica como a Revista Katálysis, Textos e Contextos, e Cadernos de Saúde Pública, indexadas na SciELO e disponibilizadas no portal Periódicos Capes.

Embora haja um número considerável de publicações em periódicos em formato eletrônico, 11 dos 14 mais utilizados para publicações, como a Revista Katálysis, o formato impresso ainda tem grande peso; os dois periódicos mais utilizados para publicações pelos pesquisadores estudados, O Social em Questão e Revista Serviço Social e Sociedade, apesar de se apresentarem em formatos impresso e eletrônico, têm na versão impressa a maioria das publicações, principalmente a analisada nesta pesquisa. Vale ressaltar que a Revista Serviço Social e Sociedade começou a divulgação online no ano de 2010, disponibilizando somente os dois últimos números em acesso aberto, e com contato para assinatura do formato impresso.

Há que se levar em consideração que as revistas em formato eletrônico são muito recentes na área e algumas, disponibilizadas nos sites das universidades editoras, ainda estão em processo de indexação, como a Libertas Online; outras não estão indexadas em bases eletrônicas nem em processo, o que torna o acesso ao seu conteúdo não tão visível na rede. Dos 11 periódicos disponibilizados eletronicamente, três não apresentavam este acesso até final de 2009 e um até meados de 2010.

\section{Indexação dos periódicos e visibilidade}

A indexação dos periódicos em base de dados foi utilizada, baseada em um dos sete indicadores de avaliação do modelo desenvolvido por Braga e Oberhofer (1982, p.28) para avaliação de periódicos brasileiros. Segundo os autores "quanto mais numerosa a inclusão em serviços de indexação, maior a disseminação". Krzyzanowski e Ferreira (1998), ao utilizarem o modelo para avaliar periódicos brasileiros, observaram que a indexação e a duração do periódico eram as duas principais variáveis para o índice desempenho.

Nesta pesquisa, a indexação em base de dados de periódicos foi o critério utilizado para analisar a visibilidade da produção científica de líderes de pesquisa em Serviço Social em conjunto com a disponibilização do texto completo via acesso aberto na Internet.

Dos 15 periódicos analisados seis estão indexados em bases de dados internacionais e nove não apresentam informações nos sites ou sistemas de editoração eletrônica. A ausência da informação sobre indexação pode corroborar a questão da pouca tradição da área em publicações periódicas que tem neste item um dos aspectos de avaliação (Figura 2). 


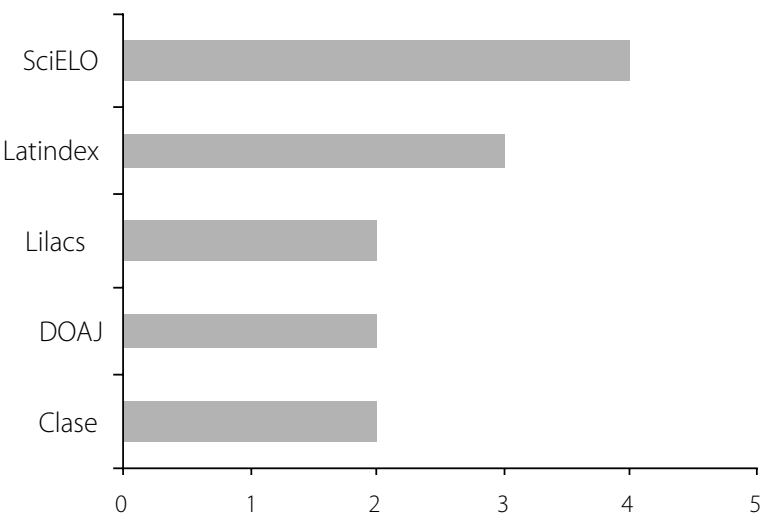

Figura 2. Principais bases de indexação dos periódicos mais utilizados para publicação da produção dos líderes de pesquisa em Serviço Social de 2003 a 2010.

As revistas da área que estão indexadas em bases de dados são Revista Katálysis, Revista Agora, Revista Textos e Contextos, Ser Social e Serviço Social e Sociedade. A primeira tem o maior número de indexações (12) dentre eles os apresentados na Figura 2 além de Redalyc (Red de Revistas Científicas de América Latina y El Caribe, España y Portugal), OEl-CREDI (Biblioteca Digital da Organização dos Estados Ibero-Americanos), IRESIE (Índice de Revistas de Educación Superior e Investigación Educativa), e portais de periódicos de acesso livre como Dialnet, LivRE!, Portal de Periódicos da Capes e Portal Periódicos UFSC. Em seguida está a Revista Agora que, além das indexações citadas na Figura 2, também está indexada na Psyclnfo (American Psychological Association) e Index Psi Revistas. A revista Textos e Contextos segue o mesmo padrão das anteriores acrescido da indexação nos Sumários de Revistas Brasileiras e disponibilização no Portal de Periódicos da Capes. A Revista mais utilizada para publicação pelo grupo estudado, Serviço Social e Sociedade, está indexada na SciELO, mas somente com os números de 2010.

O periódico Cadernos de Saúde Pública, da área da saúde apresenta o maior número de indexação (vinte) sendo a maioria da sua área específica como o Index Medicus (Medline), Abstracts on Hygiene and Communicable Diseases, RedPanamericana de Informacióny Documentación en Ingeniería Sanitaria y Ciencias del Ambiente (Repidisca); bem como bases de áreas sociais como Sociological Abstracts, Social Planning/Policy \& Development; e geral como a Scopus.

Além da presença nesses serviços, a maioria das revistas está presente nos portais das entidades edito- ras/universidades (UERJ, UFSC, UFMA) e algumas também fazem parte do Portal de Periódicos da Capes como Cadernos de Saúde Pública e Textos e Contextos.

Segundo os indicadores analisados nesta pesquisa, publicações de autores em periódicos indexados (Zimba; Mueller, 2004) e disponibilização na Internet do texto completo de (Oliveira, 2005), a produção científica dos líderes de pesquisa em Serviço Social apresenta visibilidade considerável. Contudo, vale ressaltar que o periódico mais utilizado pelos líderes estudados, Serviço Social e Sociedade, bastante representativo da área, não possibilita visibilidade desejada, uma vez que a indexação é restrita ao SciELO e somente em 2010 aderiu ao acesso livre do texto completo via Internet.

\section{Conclusão}

Considerando que diversos estudos já analisaram a importância da pesquisa em Serviço Social para a formação e intervenção profissional, ao demonstrar como o conhecimento construído ao longo das três últimas décadas do Século XX contribuiu para a transformação da realidade social e política, este estudo analisou quantitativamente a produtividade das pesquisas em Serviço Social e sua visibilidade nos canais de comunicação científica utilizados pelos líderes dos grupos de pesquisa do Estado do Rio de Janeiro no período de 2003 a 2010.

Há ainda uma acentuada tendência de publicações em periódicos híbridos - formato impresso e eletrônico. Alguns dos periódicos disponibilizados na web não são editorados eletronicamente tendo, além da versão impressa, arquivo em PDF disponibilizado no site da editora/universidade o que compromete sua recuperação na rede. Dentre os que realizam a editoração eletrônica que possibilita a recuperação dos arquivos, o SEER é o sistema mais utilizado.

Apesar de a maioria dos periódicos estar disponível na Internet, somente cinco revistas da área de Serviço Social e uma da Saúde estão indexadas em bases internacionais e portais como o Portal de Periódicos da Capes.

De forma geral, essas configurações de comunicação científica denotam uma tendência de mudança de uma área que no Brasil ainda se encontra em estágio inicial da utilização de canais formais eletrônicos. Apesar 
da ainda incipiente utilização do meio eletrônico e indexado, a velocidade da mudança no quadro merece destaque dado que no período de um ano houve mudança de impresso para eletrônico em quatro periódicos analisados.

O padrão de comunicação científica corrobora o de outras áreas das Ciências Sociais, contudo não apresenta alta visibilidade como outras áreas, principalmente Ciência da Informação, que dispõe de número expressivo de periódicos de acesso livre, além do alto número de periódicos que utilizam diversificados espaços de indexação, como bases de dados (Science Citation Index), diretórios (Latindex), bibliotecas eletrônicas (SciELO) e redes cooperativas (Redalyc).

Dessa forma, pode-se considerar que se a visibilidade da produção científica dos pesquisadores líderes de grupos do Estado do Rio de Janeiro ainda não alcançou um nível similar ao de outras áreas das Ciências Sociais Aplicadas, há indícios de mudanças na tendência de padrão de comunicação científica na área. Seria recomendável que grupos de outros Estados fossem analisados para que se pudesse ter uma compreensão maior da área de conhecimento no Brasil.

\section{Referências}

BAPTISTA, M.V. A produção do conhecimento social contemporâneo e sua ênfase no serviço social. Cadernos ABESS, v.5, p.96, 1992.

BOURGUIGNON, J.A. A particularidade histórica da pesquisa no serviço social. Revista Katálysis, v.10, n. especial, p.46-54, 2007.

BRAGA, G.; OBERHOFER C. A. Diretrizes para a avaliação de periódicos científicos e técnicos brasileiros. Revista Latina de Documentação, v.2, n.1, p.27-31, 1982.

CARVALHO, A. M. P. A pesquisa no debate contemporâneo e o serviço social. Cadernos ABESS, v.5, p.43-83, 1992.

CASTRO, R.C.F. Impacto da Internet no fluxo da comunicação científica em saúde. Revista de Saúde Pública, v.40, número Especial, p.57-63, 2006.

CHALHUB, T.; OLIVEIRA, E. P. O panorama da produção científica de pesquisadores bolsistas do CNPq em Serviço Social. In: ENCONTRO NACIONAL DE PESQUISA EM CIÊNCIA DA INFORMAÇÃO - ENANCIB 2010, 11., 2010, Rio de Janeiro. Anais... Rio de Janeiro: IBICT, 2010. Disponível em: <http:// congresso.ibict.br/index.php/enancib/xienancib/paper/view/ 537/82>. Acesso em: 12 fev. 2011.

GONZÁLEZ DE GÓMEZ, M. N.. As relações entre ciência, Estado e sociedade: um domínio de visibilidade para as questões da informação. Ciência da Informação, v.32, n.1, p.60-76, 2003. Disponível em: <http://revista.ibict.br/>. Acesso em: 15 jun. 2011.

GRUSZYNSKI, A. C.; GOLIN, C. Periódicos científicos: transição dos suportes impresso para o eletrônico e eficácia comunicacional. Unirevista, n.1, v.3, 2006.

KAMEYAMA, N. A trajetória da produção de conhecimentos em serviço social: avanços e tendências (1975 a 1997). Cadernos ABESS, v.8, p.33-76, 1998.

KRZYZANOWSKI, R.F.; FERREIRA, M.C.G. Avaliação de periódicos científicos e técnicos brasileiros. Ciência da Informação, v.27, n.2, 1998. Disponível em: <http://www.scielo.br/ scielo>. Acesso em: 28 dez. 2010.
LEITE, F. Como gerenciar e ampliar a visibilidade da informação científica brasileira: repositórios institucionais de acesso aberto. Brasília: IBICT, 2009. Disponível em: <http://www.ibict.br/ anexos_noticias/repositorios.institucionais.F.Leite_ atualizado.pdf>. Acesso em: 11 de mar. 2011.

MACHADO, R.N.; MEIRELLES, R.F. Produção científica dos docentes da Universidade Federal da Bahia da área de filosofia e ciências humanas no período de 1995-1999. Trans/nformação, v.17, n. 2, p.169-179, 2005. Disponível em: <http:// revistas.puc-campinas.edu.br/transinfo $>$. Acesso em: 11 mar. 2011.

MEADOWS, A.J. A comunicação científica. Brasília: Briquet de Lemos Livros, 1999.

OLIVEIRA, E.B. Produção científica nacional na área de geociências: análise de critérios de editoração, difusão e indexação em bases de dados. Ciência da Informação, v.34, n.2, p.34-42, 2005. Disponível em: <http://revista.ibict.br>. Acesso em: 11 fev. 2011.

OLIVERIA, E.P.; VALERIO, P.M.; CHALHUB, T.; PINHEIRO, L.V.R. Recursos eletrônicos de informação e comunicação em periódicos científicos latino-americanos em ciências agrárias. In: JORNADAS LATINOAMERICANA DE ESTUDIOS SOCIALES DE LA CIENCIA Y LA TECNOLOGIA, 8., JORNADAS LATINOAMERICANA DE ESTUDIOS SOCIALES DE LA CIENCIA Y LA TECNOLOGIA, 8., 2010, Buenos Aires. Anais... Buenos Aires: ESOCITE, 2010. v.1.

SIMIONATTO, I. Os desafios na pesquisa e na produção do conhecimento em serviço social. Temporalis, v.9, p.51-62, 2005.

SPOSATI, A. Pesquisa e produção de conhecimento no campo do serviço social. Revista Katálysis, v.10, número Especial, p.15-25, 2007. Disponível em: <http://www.scielo.br/pdf/ rk/v10nspe/a0210spe.pdf>. Acesso em: 19 dez. 2010.

YASBECK, M. C. Os caminhos para a pesquisa no serviço social. Temporalis, v.9, p 147-159, 2005. 